\title{
Simultaneous Optimal Controls for Non-Stationary Stokes Systems
}

\author{
C. M. Gariboldi ${ }^{1}$ and E. L. Schwindt ${ }^{2, *}$ \\ 1 Departamento de Matemática, Facultad de Ciencias Exactas, Físico-Químicas y \\ Naturales, Universidad Nacional de Río Cuarto, 5800-Río Cuarto, Córdoba, \\ Argentina \\ ${ }^{2}$ Laboratoire Jacques-Louis Lions, Université Pierre et Marie Curie Paris 6, UMR \\ 7598, 75005 Paris, France
}

Received 18 October 2016; Accepted (in revised version) 13 January 2017

\begin{abstract}
This paper deal with optimal control problems for a non-stationary Stokes system. We study a simultaneous distributed-boundary optimal control problem with distributed observation. We prove the existence and uniqueness of a simultaneous optimal control and we give the first order optimality condition for this problem. We also consider a distributed optimal control problem and a boundary optimal control problem and we obtain estimations between the simultaneous optimal control and the optimal controls of these last ones. Finally, some regularity results are presented.
\end{abstract}

Key Words: Simultaneous optimal controls, unsteady Stokes system, optimality condition.

AMS Subject Classifications: 49J20,76D07,65K10

\section{Introduction}

Let $\Omega$ be a bounded domain (i.e., connected and open set) of $\mathbb{R}^{3}$ with $\partial \Omega$ of class $\mathcal{C}^{2}$. We consider the following unsteady Stokes system

$$
\begin{cases}\frac{\partial y}{\partial t}-\operatorname{div} \sigma(y, p)=\boldsymbol{u} & \text { in } \Omega \times(0, T), \\ \operatorname{div} \boldsymbol{y}=0 & \text { in } \Omega \times(0, T), \\ \boldsymbol{y}=\boldsymbol{g} & \text { on } \partial \Omega \times(0, T), \\ \boldsymbol{y}(0)=\boldsymbol{a} & \text { in } \Omega .\end{cases}
$$

In this paper, we will use the notation in bold for vector functions. Here, $(y, p)=$ $\left(y_{1}, y_{2}, y_{3}, p\right)$ are the velocity and the pressure of the fluid and $\sigma(\boldsymbol{y}, p)$ denotes the Cauchy

${ }^{*}$ Corresponding author. Email addresses: cgariboldi@exa.unrc.edu.ar (C. Gariboldi), schwindt@math . cnrs.fr (E. Schwindt) 
stress tensor, which is defined by Stokes law $\sigma(\boldsymbol{y}, p)=-p \boldsymbol{I} \boldsymbol{d}+2 v \boldsymbol{D}(\boldsymbol{y})$, where $\boldsymbol{I} \boldsymbol{d}$ is the identity matrix of order $3, v$ is the kinematic viscosity of the fluid and $\boldsymbol{D}(\boldsymbol{y})$ is the strain tensor defined by

$$
[\boldsymbol{D}(\boldsymbol{y})]_{k l}=\frac{1}{2}\left(\frac{\partial y_{k}}{\partial x_{l}}+\frac{\partial y_{l}}{\partial x_{k}}\right)
$$

Since $\operatorname{div} \boldsymbol{y}=0$, we have $-\operatorname{div} \sigma(\boldsymbol{y}, p)=-v \Delta \boldsymbol{y}+\nabla p$ in $\Omega$. The System (1.1) admits a unique (up to a constant for $p$ ) solution $(\boldsymbol{y}, p)$ with

$$
(\boldsymbol{y}, p) \in L^{2}\left(0, T ; H^{1}(\Omega)\right) \cap C^{0}\left(0, T ; L^{2}(\Omega)\right) \times L^{2}\left(0, T ; L^{2}(\Omega)\right) / \mathbb{R}
$$

(see below for the notation of these spaces), provided that $u \in L^{2}\left(0, T ; L^{2}(\Omega)\right)$ and $g \in$ $L^{2}\left(0, T ; H^{1 / 2}(\partial \Omega)\right) \cap C^{0}\left(0, T ; H^{-1 / 2}(\partial \Omega)\right)$ satisfies the compatibility conditions

$$
\int_{\partial \Omega} \boldsymbol{g} \cdot \boldsymbol{n} d \gamma=0 \text { and } \boldsymbol{g}(0)=\boldsymbol{a} \text { on } \partial \Omega
$$

with $a \in H(\operatorname{div} ; \Omega)$, where

$$
H(\operatorname{div} ; \Omega)=\left\{\boldsymbol{a} \in L^{2}(\Omega) \text { such that } \operatorname{div} \boldsymbol{a}=0\right\},
$$

properties of this space can be found in [7]. Moreover, there exists a constant $K$ depending of $\Omega$ and $v$ such that

$$
\|\boldsymbol{y}\|_{L^{2}\left(H^{1}(\Omega)\right)}+\|p\|_{L^{2}\left(L^{2}(\Omega)\right)} \leq K\left(\|\boldsymbol{u}\|_{L^{2}\left(L^{2}(\Omega)\right)}+\|\boldsymbol{g}\|_{L^{2}\left(H^{1 / 2}(\partial \Omega)\right)}+\|\boldsymbol{a}\|_{L^{2}(\Omega)}\right)
$$

for results on the existence, uniqueness and regularity of solutions for Stokes equations with non homogeneous data, we refer to $[4,5,8,13,15]$.

Let $X$ be a Banach space, we will denote by $L^{p}(0, T ; X)$ the space of the all measurable functions $y$ such that $y:[0, T] \rightarrow X$ defined by $\boldsymbol{y}(t)(x)=\boldsymbol{y}(t, x)$ satisfy

$$
\begin{array}{ll}
\|\boldsymbol{y}\|_{L^{p}(0, T ; X)}=\left(\int_{0}^{T}\|\boldsymbol{y}(t)\|_{X}^{p} d t\right)^{1 / p}<+\infty, & \text { if } p \in[1,+\infty), \\
\|\boldsymbol{y}\|_{L^{\infty}(0, T ; X)}=e s S \sup _{0 \leq t \leq T}\|\boldsymbol{y}(t)\|_{X}<+\infty, & \text { if } p=+\infty .
\end{array}
$$

For the sake of simplicity, we will often use $L^{p}(X)$ instead of $L^{p}(0, T, X)$. In what follows, we will denote $(\cdot, \cdot)_{\Omega}$ and $(\cdot, \cdot) \partial \Omega$ the usual scalar products in $L^{2}\left(L^{2}(\Omega)\right)$ and $L^{2}\left(L^{2}(\partial \Omega)\right)$ respectively; and we also write $X^{*}$ the dual vectorial space of $X$ and $\langle\cdot, \cdot\rangle$ the duality pairing.

In this work we will consider $\boldsymbol{u}$ and $g$ as control variables and we fix the initial condition $\boldsymbol{a} \in H(\operatorname{div} ; \Omega)$.

Now, we formulate the optimal control problems with distributed observation that we will study in this paper. 
1. Distributed optimal control problem $\left(\mathcal{P}_{u}\right)$ : (unconstrained problem)

$$
\text { Find } \overline{\boldsymbol{u}}^{*} \in L^{2}\left(0, T ; L^{2}(\Omega)\right) \text { such that } \mathrm{J}_{1}\left(\overline{\boldsymbol{u}}^{*}\right)=\min _{\boldsymbol{u} \in L^{2}\left(L^{2}(\Omega)\right)} \mathrm{J}_{1}(\boldsymbol{u}),
$$

where $\mathrm{J}_{1}: L^{2}\left(0, T ; L^{2}(\Omega)\right) \rightarrow \mathbb{R}_{\geq 0}$ is the cost functional given by

$$
\mathrm{J}_{1}(\boldsymbol{u}):=\frac{1}{2} \int_{0}^{T} \int_{\Omega}\left|\boldsymbol{y}_{\boldsymbol{u}}-z_{d}\right|^{2} d x d t+\frac{\alpha}{2} \int_{0}^{T} \int_{\Omega}|\boldsymbol{u}|^{2} d x d t
$$

In this problem we consider $u$ as the control variable; $z_{d}$ is a given function that we assume in $L^{2}\left(L^{2}(\Omega)\right), \alpha$ is a positive constant and $y_{u}$ is the unique solution of the problem (1.1) with fixed and known $g$ and $\boldsymbol{a}$.

2. Boundary optimal control problem $\left(\mathcal{P}_{g}\right)$ :

$$
\text { Find } \overline{\boldsymbol{g}}^{*} \in \mathcal{U}_{a d} \text { such that } \mathrm{J}_{2}\left(\overline{\boldsymbol{g}}^{*}\right)=\min _{\boldsymbol{g} \in \mathcal{U}_{a d}} \mathrm{~J}_{2}(g) \text {, }
$$

where

$$
\mathcal{U}_{a d}=\left\{\boldsymbol{g} \in L^{2}\left(H^{1 / 2}(\partial \Omega)\right) \cap C^{0}\left(H^{-1 / 2}(\partial \Omega)\right): \int_{\partial \Omega} \boldsymbol{g} \cdot \boldsymbol{n}=0, \boldsymbol{g}(0)=\boldsymbol{a}\right\}
$$

and the cost functional $\mathrm{J}_{2}: \mathfrak{U}_{a d} \rightarrow \mathbb{R}_{\geq 0}$ is given by

$$
\mathrm{J}_{2}(g):=\frac{1}{2} \int_{0}^{T} \int_{\Omega}\left|y_{g}-z_{d}\right|^{2} d x d t+\frac{\beta}{2} \int_{0}^{T} \int_{\partial \Omega}|g|^{2} d \gamma d t .
$$

In this problem $g$ is considered as the control variable; $z_{d}$ is a given function that we assume in $L^{2}\left(L^{2}(\Omega)\right), \beta$ is a positive constant and $y_{g}$ is the unique solution of the problem (1.1) with fixed and known $\boldsymbol{u}$ and $\boldsymbol{a}$.

3. Simultaneous distributed-boundary optimal control problem $\left(\mathcal{P}_{\text {ug }}\right)$ :

$$
\text { Find }\left(\boldsymbol{u}^{*}, \boldsymbol{g}^{*}\right) \in \mathcal{A}_{a d} \quad \text { such that } \mathrm{J}\left(\boldsymbol{u}^{*}, \boldsymbol{g}^{*}\right)=\min _{(\boldsymbol{u}, \boldsymbol{g}) \in \mathcal{A}_{a d}} \mathrm{~J}(\boldsymbol{u}, \boldsymbol{g}), \quad\left(\mathcal{P}_{u g}\right)
$$

where $\mathcal{A}_{a d}=L^{2}\left(0, T ; L^{2}(\Omega)\right) \times \mathcal{U}_{a d}$ and the cost functional $\mathrm{J}: \mathcal{A}_{a d} \rightarrow \mathbb{R}_{\geq 0}$ is given by

$$
\mathrm{J}(\boldsymbol{u}, \boldsymbol{g}):=\frac{1}{2} \int_{0}^{T} \int_{\Omega}\left|\boldsymbol{y}_{u g}-z_{d}\right|^{2} d x d t+\frac{\alpha}{2} \int_{0}^{T} \int_{\Omega}|\boldsymbol{u}|^{2} d x d t+\frac{\beta}{2} \int_{0}^{T} \int_{\partial \Omega}|\boldsymbol{g}|^{2} d \gamma d t
$$

Here $(\boldsymbol{u}, \boldsymbol{g})$ is considered as the control variable; $z_{d}$ is a given function that we again assume in $L^{2}\left(L^{2}(\Omega)\right), \alpha$ and $\beta$ are the positive constants and $y_{u g}$ is the unique solution of the problem (1.1) with fixed and known $\boldsymbol{a}$. 
In $\mathcal{A}_{a d}$ we will consider the following norm

$$
\|(\boldsymbol{u}, \boldsymbol{g})\|_{\mathcal{A}_{a d}}^{2}=\|\boldsymbol{u}\|_{L^{2}\left(L^{2}(\Omega)\right)}^{2}+\|\boldsymbol{g}\|_{L^{2}\left(L^{2}(\partial \Omega)\right)}^{2}
$$

with this norm the space $\mathcal{A}_{a d}$ is a Hilbert space.

In a general framework, several optimal control problems of type $\left(\mathcal{P}_{u}\right)$ and $\left(\mathcal{P}_{g}\right)$ have been studied in [11]. The authors of [1-3] deal with optimal control problems for stationary Stokes and Navier-Stokes systems, in [1] the author studied a distributed control problem from a point of view theoretical and numerical and in [3] a similar study is done for a boundary control problem using another numerical method. In the case of nonstationary Stokes and Navier-Stokes equations we can quote $[9,10]$.

The goal of this paper is to study a simultaneous optimal control problem for an unsteady Stokes system. Similar optimal control problems that is tackled here are considered in [6] for steady-state mixed elliptic problems and for the parabolic problems in [16]. Exact controllability problems with simultaneous controls were introduced in [12], in contrast to our work, it is considered two or more systems of equations (coupled or not) with the controls imposed one on each system of equations and the aim is to control the solutions at some time $T>0$; while our interest is to impose simultaneous controls on the same system (1.1) and to control the solution in $\Omega \times(0, T)$.

The paper is structured as follows. In Section 2 we prove the existence and uniqueness of the solutions of the problem $\left(P_{u g}\right)$. We obtain that the cost functions $J_{1}, J_{2}$ and $J$ are Gâteaux differentiable and we give the first order optimality conditions in terms of the adjoint states of the systems. In Section 3 we get estimations between the unique solution of the problem $\left(P_{u}\right)$ and the first component of the unique solution of the problem $\left(P_{u g}\right)$. We also prove estimations between the unique solution of the problem $\left(P_{g}\right)$ and the second component of the unique solution of the problem $\left(P_{u g}\right)$. In Section 4 we give regularity results of the simultaneous optimal control and the solutions of the state and adjoint state systems. We end the paper with some comments.

\section{Results of existence and uniqueness of optimal controls}

\subsection{Distributed optimal control and boundary optimal control}

The existence and uniqueness of an optimal control $\overline{\boldsymbol{u}}^{*}$ for Problem $\left(\mathcal{P}_{u}\right)$ and an optimal control $\overline{\boldsymbol{g}}^{*}$ for Problem $\left(\mathcal{P}_{g}\right)$ follows similarly to what was done for example in [11] or [14], therefore we omit these proofs and we only recall the first order optimality conditions for them.

Theorem 2.1. There exists a unique solution $\overline{\boldsymbol{u}}^{*} \in L^{2}\left(0, T ; L^{2}(\Omega)\right)$ of the distributed optimal control problem $\left(\mathcal{P}_{u}\right)$. Furthermore, the first order optimality condition that satisfies $\overline{\boldsymbol{u}}^{*}$ can be expressed in terms of the Gâteaux derivative of its cost functional $\mathrm{J}_{1}$ as

$$
\left\langle J_{1}^{\prime}\left(\overline{\boldsymbol{u}}^{*}\right), \boldsymbol{w}\right\rangle=\left(\boldsymbol{y}_{\overline{\boldsymbol{u}}^{*}}-\boldsymbol{z}_{d}, \boldsymbol{y}_{\boldsymbol{w}}-\boldsymbol{y}_{\mathbf{0}}\right)_{\Omega}+\alpha\left(\overline{\boldsymbol{u}}^{*}, \boldsymbol{w}\right)_{\Omega}=0, \quad \forall \boldsymbol{w} \in L^{2}\left(L^{2}(\Omega)\right),
$$


where $\boldsymbol{y}_{w}-y_{0}$ satisfies (1.1) with $\boldsymbol{u}=\boldsymbol{w}, \boldsymbol{g}=\mathbf{0}$ and $\boldsymbol{a}=\mathbf{0}$. Moreover, if we introduce the adjoint state $\left(\boldsymbol{\phi}_{\bar{u}^{*}}, q_{\bar{u}^{*}}\right)$ as the unique solution of the following system:

$$
\begin{cases}-\frac{\partial \phi_{\bar{u}^{*}}}{\partial t}-\operatorname{div} \sigma\left(\phi_{\bar{u}^{*}}, q_{\bar{u}^{*}}\right)=y_{\bar{u}^{*}}-z_{d} & \text { in } \Omega \times(0, T), \\ \operatorname{div} \boldsymbol{\phi}_{\bar{u}^{*}}=0 & \text { in } \Omega \times(0, T), \\ \boldsymbol{\phi}_{\bar{u}^{*}}=\mathbf{0} & \text { on } \partial \Omega \times(0, T), \\ \boldsymbol{\phi}_{\bar{u}^{*}}(T)=\mathbf{0} & \text { in } \Omega,\end{cases}
$$

then the optimality condition given by (2.1) can be written as

$$
\left\langle J_{1}^{\prime}\left(\overline{\boldsymbol{u}}^{*}\right), \boldsymbol{w}\right\rangle=\left(\boldsymbol{\phi}_{\overline{\boldsymbol{u}}^{*}}, \boldsymbol{w}\right)_{\Omega}+\alpha\left(\overline{\boldsymbol{u}}^{*}, \boldsymbol{w}\right)_{\Omega}=0, \quad \forall \boldsymbol{w} \in L^{2}\left(L^{2}(\Omega)\right) .
$$

Theorem 2.2. There exists a unique solution $\overline{\boldsymbol{g}}^{*} \in \mathcal{U}_{a d}$ of the boundary optimal control problem $\left(\mathcal{P}_{g}\right)$. Furthermore, the first order optimality condition that satisfies $\bar{g}^{*}$ can be expressed in terms of the Gâteaux derivative of its cost functional $\mathrm{J}_{2}$ as

$$
\left\langle J_{2}^{\prime}\left(\overline{\boldsymbol{g}}^{*}\right), \boldsymbol{f}\right\rangle=\left(\boldsymbol{y}_{\overline{\boldsymbol{g}}^{*}}-\boldsymbol{z}_{d}, \boldsymbol{y}_{f}-\boldsymbol{y}_{\mathbf{0}}\right)_{\Omega}+\beta\left(\overline{\boldsymbol{g}}^{*}, \boldsymbol{f}\right)_{\partial \Omega}=0, \quad \forall \boldsymbol{f} \in \mathcal{U}_{a d},
$$

where $\boldsymbol{y}_{f}-\boldsymbol{y}_{\mathbf{0}}$ satisfies (1.1) with $\boldsymbol{u}=\mathbf{0}, \boldsymbol{g}=\boldsymbol{f}$ and $\boldsymbol{a}=\mathbf{0}$. Moreover, if we introduce the adjoint state $\left(\boldsymbol{\phi}_{\bar{g}^{*}}, q_{\bar{g}^{*}}\right)$ as the unique solution of

$$
\begin{cases}-\frac{\partial \phi_{\bar{g}^{*}}}{\partial t}-\operatorname{div} \sigma\left(\phi_{\bar{g}^{*}}, q_{\bar{g}^{*}}\right)=y_{\bar{g}^{*}}-z_{d} & \text { in } \Omega \times(0, T), \\ \operatorname{div} \boldsymbol{\phi}_{\bar{g}^{*}}=0 & \text { in } \Omega \times(0, T), \\ \boldsymbol{\phi}_{\bar{g}^{*}}=\mathbf{0} & \text { on } \partial \Omega \times(0, T), \\ \boldsymbol{\phi}_{\bar{g}^{*}}(T)=\mathbf{0} & \text { in } \Omega,\end{cases}
$$

then the optimality condition given by (2.3) can be written as

$$
\left\langle J_{2}^{\prime}\left(\overline{\boldsymbol{u}}^{*}\right), \boldsymbol{f}\right\rangle=\left(\boldsymbol{\sigma}\left(\boldsymbol{\phi}_{\overline{\boldsymbol{g}}^{*}}, q_{\overline{\boldsymbol{g}}^{*}}\right) n, \boldsymbol{f}\right)_{\partial \Omega}+\beta\left(\overline{\boldsymbol{g}}^{*}, \boldsymbol{f}\right)_{\partial \Omega}=0, \quad \forall \boldsymbol{f} \in \mathcal{U}_{a d} .
$$

\subsection{Simultaneous distributed-boundary optimal control}

We define the map

$$
\begin{aligned}
C: \mathcal{A}_{a d} & \rightarrow L^{2}\left(0, T ; H_{0}^{1}(\Omega)\right) \\
(\boldsymbol{u}, \boldsymbol{g}) & \mapsto \boldsymbol{y}_{\boldsymbol{u g}}-\boldsymbol{y}_{\mathbf{0 0}}
\end{aligned}
$$

where $y_{00}$ is the unique solution of (1.1) with $\boldsymbol{u}=\boldsymbol{g}=\mathbf{0}$ and fixed $\boldsymbol{a}$.

We also define the maps $\Pi: \mathcal{A}_{a d} \times \mathcal{A}_{a d} \rightarrow \mathbb{R}$ and $\mathrm{Y}: \mathcal{A}_{a d} \rightarrow \mathbb{R}$ as

$$
\begin{array}{ll}
\Pi((\boldsymbol{u}, \boldsymbol{g}),(\boldsymbol{v}, \boldsymbol{h}))=(C(\boldsymbol{u}, \boldsymbol{g}), C(\boldsymbol{v}, \boldsymbol{h}))_{\Omega}+\alpha(\boldsymbol{u}, \boldsymbol{v})_{\Omega}+\beta(\boldsymbol{g}, \boldsymbol{h})_{\partial \Omega}, & \forall(\boldsymbol{u}, \boldsymbol{g}),(\boldsymbol{v}, \boldsymbol{h}) \in \mathcal{A}_{a d}, \\
Y(\boldsymbol{u}, \boldsymbol{g})=\left(C(\boldsymbol{u}, \boldsymbol{g}), \boldsymbol{z}_{d}\right)_{\Omega^{\prime}} & \forall(\boldsymbol{u}, \boldsymbol{g}) \in \mathcal{A}_{a d} .
\end{array}
$$

The following properties hold for the previous maps. 
Proposition 2.1. $\quad$ i) $C$ is a linear and continuous map.

ii) $\Pi$ is a bilinear, continuous, symmetric and coercive map.

iii) $\mathrm{Y}$ is a linear and continuous map.

Proof. i) The linearity follows immediately from the definition of $C$. Taking into account (1.2)-(1.3) we deduce that, in particular, $\left(\boldsymbol{y}_{u g}-y_{00}, p_{u g}-p_{00}\right) \in L^{2}\left(H^{1}(\Omega)\right) \times L^{2}\left(L^{2}(\Omega)\right) / \mathbb{R}$ and it satisfies

$$
\|C(\boldsymbol{u}, \boldsymbol{g})\|_{L^{2}\left(H^{1}(\Omega)\right)}=\left\|\boldsymbol{y}_{\boldsymbol{u g}}-\boldsymbol{y}_{\mathbf{0 0}}\right\|_{L^{2}\left(H^{1}(\Omega)\right)} \leq K_{1}\|(\boldsymbol{u}, \boldsymbol{g})\|_{\mathcal{A}_{\text {ad }}}
$$

where $K_{1}$ is a constant depending on the viscosity $v$ and the domain $\Omega$, thus the continuity of $C$ is proved.

ii) The bilinearity of $\Pi$ follows from the linearity of $C$. From Cauchy-Schwartz inequality and the continuity of $C$ we obtain the continuity of $\Pi$, indeed $\forall(\boldsymbol{u}, \boldsymbol{g}),(\boldsymbol{v}, \boldsymbol{h}) \in \mathcal{A}_{a d}$

$$
|\Pi((\boldsymbol{u}, \boldsymbol{g}),(\boldsymbol{v}, \boldsymbol{h}))| \leq K_{2}\|(\boldsymbol{u}, \boldsymbol{g})\|_{\mathcal{A}_{a d}}\|(\boldsymbol{v}, \boldsymbol{h})\|_{\mathcal{A}_{a d}},
$$

where $K_{2}$ is a constant depending on $K_{1}, \alpha$ and $\beta$. Moreover, $\Pi$ is coercive, in effect

$$
|\Pi((\boldsymbol{u}, \boldsymbol{g}),(\boldsymbol{u}, \boldsymbol{g}))| \geq \alpha\|\boldsymbol{u}\|_{L^{2}\left(L^{2}(\Omega)\right)}+\beta\|\boldsymbol{g}\|_{L^{2}\left(L^{2}(\partial \Omega)\right)} \geq K_{3}\|(\boldsymbol{u}, \boldsymbol{g})\|_{\mathcal{A}_{a d^{\prime}}}^{2}
$$

where $K_{3}$ depends on $\alpha$ and $\beta$.

iii) It follows from the linearity and continuity of $C$.

After some calculations, it is possible to rewrite the cost functional $J$ as

$$
J(\boldsymbol{u}, \boldsymbol{g})=\frac{1}{2} \Pi((\boldsymbol{u}, \boldsymbol{g}),(\boldsymbol{u}, \boldsymbol{g}))-Y(\boldsymbol{u}, \boldsymbol{g})+\frac{1}{2}\left\|z_{d}\right\|_{L^{2}\left(L^{2}(\Omega)\right)}^{2} .
$$

Theorem 2.3 (Existence and uniqueness). There exists a unique solution $\left(\boldsymbol{u}^{*}, \boldsymbol{g}^{*}\right) \in \mathcal{A}_{a d}$ of the simultaneous distributed-boundary optimal control problem $\left(\mathcal{P}_{u g}\right)$.

Proof. Taking into account (2.5) and Proposition 2.1 we can deduce that $J$ is a strictly convex function. Thus, by the classical theory of optimal control [11, Chapter 3], we have that there exists a unique solution $\left(\boldsymbol{u}^{*}, \boldsymbol{g}^{*}\right) \in \mathcal{A}_{a d}$ of the problem $\left(\mathcal{P}_{u g}\right)$.

Now, we compute the derivative of the cost functional $J$.

Proposition 2.2. The function $J$ is Gâteaux differentiable and its derivate $J^{\prime}: \mathcal{A}_{a d} \rightarrow\left[\mathcal{A}_{a d}\right]^{*}$ at $(\boldsymbol{u}, \boldsymbol{g})$ is given by

$$
\left\langle J^{\prime}(\boldsymbol{u}, \boldsymbol{g}),(\boldsymbol{v}, \boldsymbol{h})\right\rangle=\left(\boldsymbol{y}_{u \boldsymbol{g}}-\boldsymbol{z}_{d}, \boldsymbol{y}_{\boldsymbol{v} h}-\boldsymbol{y}_{\mathbf{0 0}}\right)_{\Omega}+\alpha(\boldsymbol{u}, \boldsymbol{v})_{\Omega}+\beta(\boldsymbol{g}, \boldsymbol{h})_{\partial \Omega}, \quad \forall(\boldsymbol{v}, \boldsymbol{h}) \in \mathcal{A}_{a d} .
$$

We note that $\boldsymbol{y}_{\boldsymbol{v}}-\boldsymbol{y}_{\mathbf{0 0}}$ satisfies (1.1) with $\boldsymbol{u}=\boldsymbol{v}, \boldsymbol{g}=\boldsymbol{h}$ and $\boldsymbol{a}=\mathbf{0}$. 
Proof. For all $(\boldsymbol{v}, \boldsymbol{h}) \in \mathcal{A}_{a d}$ and $t>0$ we have

$$
\begin{aligned}
\frac{1}{t}[J(\boldsymbol{u}+t \boldsymbol{v}, \boldsymbol{g}+t \boldsymbol{h})-J(\boldsymbol{u}, \boldsymbol{g})]= & \left(\boldsymbol{y}_{\boldsymbol{u g}}-\boldsymbol{z}_{d}, \boldsymbol{y}_{v \boldsymbol{h}}-\boldsymbol{y}_{\mathbf{0 0}}\right)_{\Omega}+\frac{t}{2}\left(\boldsymbol{y}_{v \boldsymbol{h}}-\boldsymbol{y}_{00}, \boldsymbol{y}_{v \boldsymbol{h}}-\boldsymbol{y}_{00}\right)_{\Omega} \\
& +\alpha(\boldsymbol{u}, \boldsymbol{v})_{\Omega}+\frac{t}{2} \alpha(\boldsymbol{v}, \boldsymbol{v})_{\Omega}+\beta(\boldsymbol{g}, \boldsymbol{h})_{\partial \Omega}+\frac{t}{2} \beta(\boldsymbol{h}, \boldsymbol{h})_{\partial \Omega}
\end{aligned}
$$

and passing to the limit $t \rightarrow 0^{+}$, we obtain (2.6).

Thanks to Proposition 2.2 we can give the first order optimality condition for $\left(\boldsymbol{u}^{*}, \boldsymbol{g}^{*}\right)$. Namely, the unique solution $\left(\boldsymbol{u}^{*}, \boldsymbol{g}^{*}\right)$ of $\left(\mathcal{P}_{u g}\right)$ can be characterized as the unique pair of functions in $\mathcal{A}_{a d}$ that satisfies

$$
\left\langle J^{\prime}\left(\boldsymbol{u}^{*}, \boldsymbol{g}^{*}\right),(\boldsymbol{v}, \boldsymbol{h})\right\rangle=\left(\boldsymbol{y}_{\boldsymbol{u}^{*} \boldsymbol{g}^{*}}-\boldsymbol{z}_{d}, \boldsymbol{y}_{\boldsymbol{v} \boldsymbol{h}}-\boldsymbol{y}_{\mathbf{0 0}}\right)_{\Omega}+\alpha\left(\boldsymbol{u}^{*}, \boldsymbol{v}\right)_{\Omega}+\beta\left(\boldsymbol{g}^{*}, \boldsymbol{h}\right)_{\partial \Omega}=0
$$

for all $(v, h) \in \mathcal{A}_{a d}$.

Since Eq. (2.7) does not permit to express the first order optimality condition easily, we introduce the adjoint state to rewrite this derivative into a more workable expression. For this, let us consider the following system

$$
\begin{cases}-\frac{\partial}{\partial t} \phi_{u^{*} g^{*}}-\operatorname{div} \sigma\left(\phi_{u^{*} g^{*}}, q_{u^{*} g^{*}}\right)=y_{u^{*} g^{*}}-z_{d} & \text { in } \Omega \times(0, T), \\ \operatorname{div} \phi_{u^{*} g^{*}}=0 & \text { in } \Omega \times(0, T), \\ \phi_{u^{*} g^{*}}=\mathbf{0} & \text { on } \partial \Omega \times(0, T), \\ \phi_{u^{*} g^{*}}(T)=\mathbf{0} & \text { in } \Omega .\end{cases}
$$

After the change of variable $(t, x) \mapsto(T-t, x)$, from the regularity $y_{u^{*} g^{*}}-z_{d} \in$ $L^{2}\left(0, T ; L^{2}(\Omega)\right)$, the above system has a unique solution $\left(\phi_{u^{*} g^{*}}, q_{u^{*}} g^{*}\right)$ such that

$$
\left(\boldsymbol{\phi}_{u^{*} g^{*}}, q_{u^{*} g^{*}}\right) \in L^{2}\left(0, T ; H^{2}(\Omega)\right) \cap C^{0}\left(0, T ; H^{1}(\Omega)\right) \times L^{2}\left(0, T ; H^{1}(\Omega)\right) / \mathbb{R} .
$$

Moreover, there exists a constant $K$ depends only of $\Omega$ and $v$ such that

$$
\left\|\boldsymbol{\phi}_{\boldsymbol{u}^{*} \boldsymbol{g}^{*}}\right\|_{L^{2}\left(H^{2}(\Omega)\right)}+\left\|q_{\boldsymbol{u}^{*} \boldsymbol{g}^{*}}\right\|_{L^{2}\left(H^{1}(\Omega)\right)} \leq K\left\|\boldsymbol{y}_{\boldsymbol{u}^{*} \boldsymbol{g}^{*}}-\boldsymbol{z}_{d}\right\|_{L^{2}\left(L^{2}(\Omega)\right)} .
$$

Now, we are in position to establish the following result

Proposition 2.3. The first order optimality condition (2.7) can be written in terms of the adjoint state as

$$
\begin{aligned}
& \left\langle J^{\prime}\left(\boldsymbol{u}^{*}, \boldsymbol{g}^{*}\right),(\boldsymbol{v}, \boldsymbol{h})\right\rangle \\
= & \left(\boldsymbol{\phi}_{\boldsymbol{u}^{*} \boldsymbol{g}^{*}}+\alpha \boldsymbol{u}^{*}, \boldsymbol{v}\right)_{\Omega}+\left(\beta \boldsymbol{g}^{*}-\boldsymbol{\sigma}\left(\boldsymbol{\phi}_{\boldsymbol{u}^{*} \boldsymbol{g}^{*}}, \boldsymbol{q}_{\boldsymbol{u}^{*} \boldsymbol{g}^{*}}\right) \boldsymbol{n}, \boldsymbol{h}\right)_{\partial \Omega}=0, \quad \forall(\boldsymbol{v}, \boldsymbol{h}) \in \mathcal{A}_{a d},
\end{aligned}
$$

and the simultaneous optimal control $\left(\boldsymbol{u}^{*}, \boldsymbol{g}^{*}\right)$ is given by

$$
\boldsymbol{u}^{*}=\frac{-1}{\alpha} \boldsymbol{\phi}_{\boldsymbol{u}^{*} \boldsymbol{g}^{*}} \quad \text { in } \Omega \times(0, T) \quad \text { and } \quad \boldsymbol{g}^{*}=\frac{1}{\beta} \sigma\left(\boldsymbol{\phi}_{\boldsymbol{u}^{*} \boldsymbol{g}^{*}}, q_{\boldsymbol{u}^{*} \boldsymbol{g}^{*}}\right) \boldsymbol{n} \quad \text { on } \partial \Omega \times(0, T) \text {. }
$$


Proof. By using (2.8), (2.7) and integrating by parts, we have

$$
\left(\boldsymbol{y}_{u^{*} g^{*}}-z_{d}, \boldsymbol{y}_{\boldsymbol{v} h}-\boldsymbol{y}_{00}\right)_{\Omega}=\left(\boldsymbol{\phi}_{u^{*} g^{*}}, \boldsymbol{v}\right)_{\Omega}-\left(\sigma\left(\boldsymbol{\phi}_{u^{*} g^{*}}, q_{u^{*} g^{*}}\right) \boldsymbol{n}, \boldsymbol{h}\right)_{\partial \Omega}, \quad \forall(\boldsymbol{v}, \boldsymbol{h}) \in \mathcal{A}_{a d},
$$

therefore we obtain (2.11). The equalities (2.12) follows by decoupling (2.11).

The following theorem summarizes the optimality system for Problem $\left(\mathcal{P}_{u g}\right)$.

Theorem 2.4. Let $\left(\boldsymbol{u}^{*}, \boldsymbol{g}^{*}\right)$ be the unique optimal solution to Problem $\left(\mathcal{P}_{u g}\right)$. Then,

- the state equation (1.1) is satisfied,

- the adjoint equation (2.8) is satisfied,

- equality (2.11) holds.

\section{Estimations}

In this section, we obtain estimations between the simultaneous optimal control and the distributed optimal control and boundary optimal control respectively.

Proposition 3.1. Let $\left(\boldsymbol{u}^{*}, \boldsymbol{g}^{*}\right)$ be the unique solution of the optimal control problem $\left(\mathcal{P}_{u g}\right)$ with fixed given initial condition $a$.

i) If $\overline{\boldsymbol{u}}^{*}$ is the unique solution of the optimal control problem $\left(\mathcal{P}_{u}\right)$ for a function fixed $g \in \mathcal{U}_{a d}$, then

$$
\left\|\boldsymbol{u}^{*}-\overline{\boldsymbol{u}}^{*}\right\|_{L^{2}\left(L^{2}(\Omega)\right)} \leq \frac{1}{\alpha}\left\|\boldsymbol{\phi}_{\boldsymbol{u}^{*} \boldsymbol{g}^{*}}-\boldsymbol{\phi}_{\overline{\boldsymbol{u}}^{*}}\right\|_{L^{2}\left(L^{2}(\Omega)\right)} \leq \frac{K}{\alpha}\left\|\boldsymbol{y}_{\boldsymbol{u}^{*} \boldsymbol{g}^{*}}-\boldsymbol{y}_{\overline{\boldsymbol{u}}^{*}}\right\|_{L^{2}\left(L^{2}(\Omega)\right)} .
$$

ii) If $\overline{\boldsymbol{g}}^{*}$ is the unique solution of the optimal control problem $\left(\mathcal{P}_{g}\right)$ for a function fixed $\boldsymbol{u} \in L^{2}\left(L^{2}(\Omega)\right)$, then

$$
\left\|\boldsymbol{g}^{*}-\overline{\boldsymbol{g}}^{*}\right\|_{L^{2}\left(L^{2}(\partial \Omega)\right)} \leq \frac{1}{\beta}\left\|\boldsymbol{\phi}_{\boldsymbol{u}^{*} \boldsymbol{g}^{*}}-\boldsymbol{\phi}_{\overline{\boldsymbol{g}}^{*}}\right\|_{L^{2}\left(L^{2}(\Omega)\right)} \leq \frac{K}{\beta}\left\|\boldsymbol{y}_{\boldsymbol{u}^{*} \boldsymbol{g}^{*}}-\boldsymbol{y}_{\overline{\boldsymbol{g}}^{*}}\right\|_{L^{2}\left(L^{2}(\Omega)\right)} .
$$

Here $K$ denote a positive constant depending on $\Omega$ and $v$.

Proof. i) Taking $(\boldsymbol{v}, \boldsymbol{h})=\left(\overline{\boldsymbol{u}}^{*}-\boldsymbol{u}^{*}, \mathbf{0}\right)$ in (2.11) and $\boldsymbol{w}=\boldsymbol{u}^{*}-\overline{\boldsymbol{u}}^{*}$ in (2.2) we have

$$
\left(\boldsymbol{\phi}_{\boldsymbol{u}^{*} g^{*}}+\alpha \boldsymbol{u}^{*}, \overline{\boldsymbol{u}}^{*}-\boldsymbol{u}^{*}\right)_{\Omega}-\left(\boldsymbol{\phi}_{\overline{\boldsymbol{u}}^{*}}+\alpha \overline{\boldsymbol{u}}^{*}, \overline{\boldsymbol{u}}^{*}-\boldsymbol{u}^{*}\right)_{\Omega}=0 .
$$

Using Cauchy-Schwartz inequality, we obtain

$$
\left\|\boldsymbol{u}^{*}-\overline{\boldsymbol{u}}^{*}\right\|_{L^{2}\left(L^{2}(\Omega)\right)} \leq \frac{1}{\alpha}\left\|\boldsymbol{\phi}_{\boldsymbol{u}^{*} \boldsymbol{g}^{*}}-\boldsymbol{\phi}_{\overline{\boldsymbol{u}}^{*}}\right\|_{L^{2}\left(L^{2}(\Omega)\right)} .
$$


Now, $\left(\boldsymbol{\phi}_{u^{*} g^{*}}-\boldsymbol{\phi}_{\bar{u}^{*}}, q_{u^{*} g^{*}}-q_{\bar{u}^{*}}\right)$ satisfies (2.8) with second membre $\boldsymbol{y}_{u^{*} g^{*}}-\boldsymbol{y}_{\bar{u}^{*}}$, thus the second inequality of $i$ ) follows from (2.10).

ii) Similarly, taking $(\boldsymbol{v}, \boldsymbol{h})=\left(\mathbf{0}, \overline{\boldsymbol{g}}^{*}-\boldsymbol{g}^{*}\right)$ in (2.11) and $f=\boldsymbol{g}^{*}-\overline{\boldsymbol{g}}^{*}$ in (2.4) we have

$$
\left(\phi_{u^{*} g^{*}}+\beta \boldsymbol{g}^{*}, \overline{\boldsymbol{g}}^{*}-\boldsymbol{g}^{*}\right)_{\partial \Omega}-\left(\boldsymbol{\phi}_{\overline{\boldsymbol{u}}^{*}}+\beta \overline{\boldsymbol{g}}^{*}, \overline{\boldsymbol{g}}^{*}-\boldsymbol{g}^{*}\right)_{\partial \Omega}=0 .
$$

Using Cauchy-Schwartz inequality, we obtain

$$
\left\|\boldsymbol{g}^{*}-\overline{\boldsymbol{g}}^{*}\right\|_{L^{2}\left(L^{2}(\partial \Omega)\right)} \leq \frac{1}{\beta}\left\|\boldsymbol{\phi}_{\boldsymbol{u}^{*} \boldsymbol{g}^{*}}-\boldsymbol{\phi}_{\overline{\boldsymbol{u}}^{*}}\right\|_{L^{2}\left(L^{2}(\Omega)\right)} .
$$

The second inequality of ii) follows also from (2.10).

Corollary 3.1. Let $\left(\boldsymbol{u}^{*}, \boldsymbol{g}^{*}\right)$ be the unique solution of Problem $\left(\mathcal{P}_{u g}\right)$. We consider $\overline{\boldsymbol{u}}^{*}$ the unique solution of Problem $\left(\mathcal{P}_{u}\right)$ with fixed boundary data $g=g^{*}$ and $\bar{g}^{*}$ the unique solution of Problem $\left(\mathcal{P}_{g}\right)$ with fixed data $\boldsymbol{u}=\boldsymbol{u}^{*}$. Then, $\overline{\boldsymbol{u}}^{*}=\boldsymbol{u}^{*}$ and $\overline{\boldsymbol{g}}^{*}=\boldsymbol{g}^{*}$.

Proof. If we take $\boldsymbol{w}=\boldsymbol{u}^{*}-\overline{\boldsymbol{u}}^{*} \in L^{2}\left(L^{2}(\Omega)\right)$ in (2.2) for $\overline{\boldsymbol{g}}^{*}=\boldsymbol{g}^{*}$ and $(\boldsymbol{v}, \boldsymbol{h})=\left(\overline{\boldsymbol{u}}^{*}-\boldsymbol{u}^{*}, \mathbf{0}\right) \in \mathcal{A}_{a d}$ in (2.11), we obtain

$$
\left(\boldsymbol{\phi}_{\overline{\boldsymbol{u}}^{*} \boldsymbol{g}^{*}}-\boldsymbol{\phi}_{\boldsymbol{u}^{*} \boldsymbol{g}^{*}}, \boldsymbol{u}^{*}-\overline{\boldsymbol{u}}^{*}\right)_{\Omega}-\alpha\left(\boldsymbol{u}^{*}-\overline{\boldsymbol{u}}^{*}, \boldsymbol{u}^{*}-\overline{\boldsymbol{u}}^{*}\right)_{\Omega}=0,
$$

taking into account that

$$
\left(\phi_{\bar{u}^{*} g^{*}}-\phi_{u^{*} g^{*}}, u^{*}-\bar{u}^{*}\right)_{\Omega}=-\left(y_{u^{*} g^{*}}-y_{\bar{g}^{*} g^{*}}, y_{u^{*} g^{*}}-y_{\bar{g}^{*} g^{*}}\right)_{\Omega}
$$

we obtain that $\overline{\boldsymbol{u}}^{*}=\boldsymbol{u}^{*}$.

By a similar argument we can prove that $\bar{g}^{*}=g^{*}$.

\section{Regularity of solutions of the optimality system}

In this section we discuss about the regularity of the simultaneous optimal control and the regularity of the solutions of the state and adjoint equations.

Proposition 4.1 (Higher regularity of the optimal control). Let $\left(\boldsymbol{u}^{*}, \boldsymbol{g}^{*}\right) \in \mathcal{A}_{\text {ad }}$ the simultaneous optimal control given by Theorem 2.3 , then actually $\left(\boldsymbol{u}^{*}, \boldsymbol{g}^{*}\right) \in L^{2}\left(0, T ; H^{2}(\Omega)\right) \cap$ $C^{0}\left(0, T ; H^{1}(\Omega)\right) \times \mathcal{U}_{a d}$. Furthermore, if $\partial \Omega \in C^{3}$ and $z_{d} \in L^{2}\left(0, T ; H^{1}(\Omega)\right)$ we have

$$
\left(\boldsymbol{u}^{*}, \boldsymbol{g}^{*}\right) \in L^{2}\left(0, T ; H^{3}(\Omega)\right) \cap C^{0}\left(0, T ; H^{2}(\Omega)\right) \times L^{2}\left(0, T ; H^{3 / 2}(\Omega)\right) \cap C^{0}\left(0, T ; H^{1 / 2}(\Omega)\right) .
$$

Proof. Since $\left(\boldsymbol{u}^{*}, \boldsymbol{g}^{*}\right) \in \mathcal{A}_{a d}$, from the regularity of the solution $\left(\boldsymbol{\phi}_{\boldsymbol{u}^{*} g^{*}}, q_{\boldsymbol{u}^{*} \boldsymbol{g}^{*}}\right)$ given by (2.9) and from (2.12) we deduce

$$
\left(\boldsymbol{u}^{*}, \boldsymbol{g}^{*}\right) \in L^{2}\left(H^{2}(\Omega)\right) \cap C^{0}\left(H^{1}(\Omega)\right) \times \mathcal{U}_{a d} .
$$


We recall that the search for $\boldsymbol{u}^{*}$ was originally in $L^{2}\left(L^{2}(\Omega)\right)$. However we can not improve the regularity of $g^{*}$, this drawback is due to the characterization "type Newmann" we get for $g^{*}$ (see (2.12)). We need to assume that the boundary $\partial \Omega \in C^{3}$ and the known wanted solution $z_{d} \in L^{2}\left(H^{1}(\Omega)\right)$ to obtain a higher regularity result for $g^{*}$, under these hypothesis we have

$$
\left(\boldsymbol{\phi}_{\boldsymbol{u}^{*} \boldsymbol{g}^{*}}, q_{\boldsymbol{u}^{*} \boldsymbol{g}^{*}}\right) \in L^{2}\left(0, T ; H^{3}(\Omega)\right) \cap C^{0}\left(0, T ; H^{2}(\Omega)\right) \times L^{2}\left(0, T ; H^{2}(\Omega)\right) / \mathbb{R},
$$

hence, from (2.12) we deduce (4.1).

The regularity result obtained for the simultaneous optimal control, in the last proposition, improves the regularity of the solution $\left(\boldsymbol{y}_{u^{*} g^{*}}, p_{u^{*} g^{*}}\right)$ of state equation.

Proposition 4.2 (Higher regularity of the solutions of the state equations). Let $\left(\boldsymbol{u}^{*}, \boldsymbol{g}^{*}\right) \in$ $\mathcal{A}_{a d}$ the simultaneous optimal control given by Theorem 2.3 , we assume that $\partial \Omega \in C^{3}$, $\boldsymbol{z}_{d} \in L^{2}\left(0, T ; H^{1}(\Omega)\right)$ and $\boldsymbol{a} \in H(\operatorname{div} ; \Omega) \cap H^{1}(\Omega)$. Then

$$
\left(\boldsymbol{y}_{\boldsymbol{u}^{*} \boldsymbol{g}^{*}}, \boldsymbol{p}_{\boldsymbol{u}^{*} \boldsymbol{g}^{*}}\right) \in L^{2}\left(0, T ; H^{2}(\Omega)\right) \cap C^{0}\left(0, T ; H^{1}(\Omega)\right) \times L^{2}\left(0, T ; H^{1}(\Omega)\right) / \mathbb{R} .
$$

Proof. Under these assumptions we can apply Proposition 4.1 to obtain (4.1). From the regularity results for the solutions of non-stationary Stokes systems with non homogeneous data (see $[4,5,8,13,15])$, we deduce (4.3).

Remark 4.1. We note that under assumptions of Proposition 4.2 and (4.3), the regularity of $\left(\phi_{u^{*} g^{*}}, q_{u^{*} g^{*}}\right)$ in not better than (4.2), since the right side of (2.8) belongs still to $L^{2}\left(0, T ; H^{1}(\Omega)\right)$ because the hypothesis on $z_{d}$. To improve (4.2) we need to assume $\partial \Omega \in C^{4}$ and $z_{d} \in L^{2}\left(0, T ; H^{2}(\Omega)\right)$.

\section{Comments}

We want to remark that the results of Sections 1-3 could be obtained if we make the assumption that the control $\boldsymbol{u}$ only acts on a non empty open subset $\mathcal{O} \subset \Omega$ and $\boldsymbol{g}$ only acts on a non empty open subset $\Gamma \subset \partial \Omega$, that is if we consider the system

$$
\begin{cases}\frac{\partial y}{\partial t}-\operatorname{div} \sigma(y, p)=\boldsymbol{F}+\mathbb{1}_{\mathcal{O}} \boldsymbol{u} & \text { in } \Omega \times(0, T), \\ \operatorname{div} \boldsymbol{y}=0 & \text { in } \Omega \times(0, T), \\ \boldsymbol{y}=\boldsymbol{H}+\mathbb{1}_{\Gamma} \boldsymbol{g} & \text { on } \partial \Omega \times(0, T), \\ \boldsymbol{y}(0)=\boldsymbol{a} & \text { in } \Omega,\end{cases}
$$

where $\boldsymbol{F} \in L^{2}\left(0, T ; L^{2}(\Omega)\right), \boldsymbol{H} \in \mathcal{U}_{a d}, \mathbb{1}_{\mathcal{O}}$ and $\mathbb{1}_{\Gamma}$ denote the characteristic functions of the subsets $\mathcal{O}$ and $\Gamma$ respectively and $\boldsymbol{a}, \boldsymbol{u}$ and $\boldsymbol{g}$ as before.

The results of Section 4 also remains true but under higher regularity assumptions on $\boldsymbol{F}$ and $\boldsymbol{H}$. 


\section{Acknowledgments}

The work of C. Gariboldi was partially supported by PIP No. 0534 from CONICET-Univ. Austral and PPI No. 18C417 from SECyT-UNRC. The work of E. Schwindt was partially supported by AVENTURES-ANR-12-BLAN-BS01-0001-01 and part of it was realized during my stay at the Institut Elie Cartan de Lorraine.

\section{References}

[1] J. C. De los Reyes, A primal-dual active set method for bilaterally control constrained optimal control of the Navier-Stokes equations, Numer. Functional Anal. Optim., 25 (2004), 657-683.

[2] J. C. De los Reyes and R. Griesse, State-constrained optimal control of the three-dimensional stationary Navier-Stokes equations, J. Math. Anal. Appl., 343 (2008), 257-272.

[3] J. C. De los Reyes and K. Kunisch, A semi-smooth Newton method for control constrained boundary optimal control of the Navier-Stokes equations, Nonlinear Anal. Theor. Methods Appl. Series A Theory Methods, 62 (2005), 1289-1316.

[4] R. Farwig, G. P. Galdi and H. Sohr, A new class of weak solutions of the Navier-Stokes equations with nonhomogeneous data, J. Math. Fluid Mech., 8 (2006), 423-444.

[5] A. V. Fursikov, M. D. Gunzburger and L. S. Hou, Inhomogeneous boundary value problem$\mathrm{s}$ for the three-dimensional evolutionary Navier-Stokes equations, J. Math. Fluid Mech., 4 (2002), 45-75.

[6] C. M. Gariboldi and D. A. Tarzia, Existence, uniqueness and convergence of simultaneous distributed-boundary optimal control problems, Control Cybernetics, 44 (2015), 5-17.

[7] V. Girault and P. A. Raviart, Finite Element Approximation of the Navier-Stokes Equations, Lecture Notes in Mathematics, Springer-Verlag, Berlin-New York, 1979.

[8] G. Grubb, Nonhomogeneous Dirichlet Navier-Stokes problems in low regularity $L_{p}$ Sobolev spaces, J. Math. Fluid Mech., 3 (2001), 57-81.

[9] M. Hintermüller, K. Kunisch, Y. Spasov and S. Volkwein, Dynamical systems-based optimal control of incompressible fluids, Int. J. Numer. Methods Fluids, 46 (2004), 345-359.

[10] M. Hinze and K. Kunisch, Second order methods for boundary control of the instationary Navier-Stokes system, ZAMM. Zeitschrift für Angewandte Mathematik und Mechanik, 84 (2004), 171-187.

[11] J. L. Lions, Contrôle Optimal de Systèmes Gouvernés par des Équations aux Dérivées Partielles, Avant propos de P. Lelong, Dunod, Paris; Gauthier-Villars, Paris, 1968.

[12] J. L. Lions, Contrôlabilité Exacte, Perturbations et Stabilisation de Systèmes Distribués, Recherches en Mathématiques Appliquées [Research in Applied Mathematics], Masson, Paris, 1988.

[13] J. L. Lions and E. Magenes, Problèmes aux Limites non Homogènes et Applications, Travaux et Recherches Mathématiques, No. 17, Dunod, Paris, 1968-1970.

[14] J. L. Menaldi and D. A. Tarzia, A distributed parabolic control with mixed boundary conditions, Asymp. Anal., 52 (2007), 227-241.

[15] H. Sohr, The Navier-Stokes Equations, Modern Birkhäuser Classics, Birkhäuser/Springer Basel AG, Basel, 2001.

[16] F. Tröltzsch, Optimal Control of Partial Differential Equations, Graduate Studies in Mathematics, American Mathematical Society, Providence, RI, 2010. 